Resaerch Paper

\title{
Reprogramming by Cytosolic Extract of Human Embryonic Stem Cells to Improve Dopaminergic Differentiation Potential of Human Adipose Tissue-derived Stem Cells
}

\author{
Sehar Mobasseri ${ }^{1}$, Arash Javeri², Masoumeh Fakhr Taha ${ }^{3^{*}}$ (i) \\ 1 Department of Medical Biotechnology, National Institute of Genetic Engineering and Biotechnology, Tehran, Iran.
}

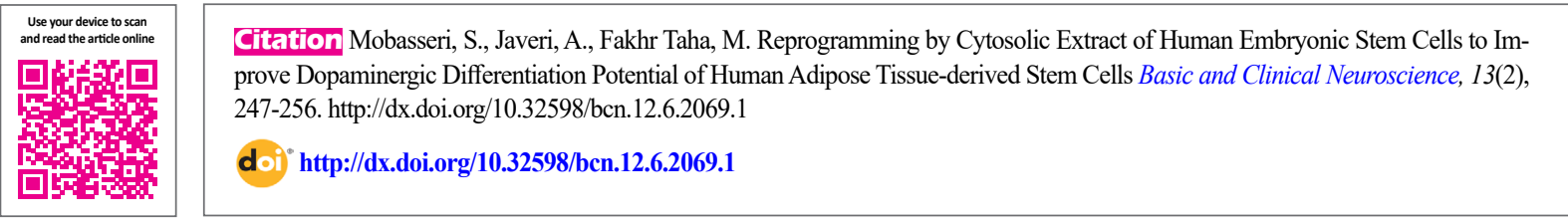

\section{(i) (8)}

Article info:

Received: 23 Sep 2019

First Revision: 13 Jun 2020

Accepted: 25 Aug 2020

Available Online: 01 Mar 2022

Keywords:

Embryonic stem cells, Adipose tissue-derived stem cells, Cytoplasmic extract, Reprogramming, Dopaminergic differentiation

\begin{abstract}
AB S T RAC T
Introduction: The extract of pluripotent stem cells induces dedifferentiation of somatic cells with restricted plasticity.

Methods: In this study, we used the extract of human embryonic stem cells (hESC) to dedifferentiate adipose tissue-derived stem cells (ADSCs) and examined the impact of this reprogramming event on the dopaminergic differentiation of the cells. For this purpose, cytoplasmic extract of ESCs was prepared by repeated freezing and thawing cycles. The plasma membrane of hADSCs was reversibly permeabilized by streptolysin O (SLO), exposed to $\mathrm{hESC}$ extract, and resealed by a $\mathrm{CaCl} 2$-containing medium.

Results: As revealed by qPCR analysis, expression of OCT4, SOX2, NANOG, LIN28A, and KLF4 mRNAs were downregulated in the ADSCs one week after extract incubation, while all mRNAs except for KLF4 were upregulated at the end of the second week. For dopaminergic differentiation, control and reprogrammed ADSCs were induced by a serum-free neurobasal medium containing B27 and a cocktail of sonic hedgehog $(\mathrm{SHH})$, basic fibroblast growth factor (bFGF), fibroblastic growth factor 8 (FGF8), and brain-derived neurotrophic factor (BDNF) for 12 days. After differentiation, the expression levels of some neuronal and dopaminergicrelated genes, including PAX6, NESTIN, NEFL, GLI1, LMXB1, EN1, NURR1, and TH, significantly increased in the reprogrammed ADSCs compared to the control group. On the whole, two weeks after reprogramming by ESC extract, ADSCs showed an improved dopaminergic differentiation potential.

Conclusion: These findings suggest that the cytoplasmic extract of hESCs contains some regulatory factors which induce the expression of pluripotency-associated markers in somatic cells and that the exposure to ESC extract may serve as a simple and rapid strategy to enhance the plasticity of somatic stem cells for cell replacement therapy purposes.
\end{abstract}

\footnotetext{
* Corresponding Author: Masoumeh Fakhr Taha, PhD.

Address: Department of Medical Biotechnology, National Institute of Genetic Engineering and Biotechnology, Tehran, Iran. Tel: +98 (21) 44580381

E-mail: mftaha@nigeb.ac.ir
} 


\section{Highlights}

- hADSCs have emerged as a valuable candidate for transplantation therapy of neurodegenerative diseases.

- Several studies have documented dopaminergic dedifferentiation of hADSCs.

- Implementing ADSCs towards a more pluripotent state using different strategies like somatic cell nuclear transfer

\section{Plain Language Summary}

The extract of pluripotent stem cells induces dedifferentiation of somatic cells with restricted plasticity. In this study, we used the extract of hESC to dedifferentiate ADSCs and examined the impact of this reprogramming event on the dopaminergic differentiation of the cells. Cytoplasmic extract of ESCs was prepared by repeated freezing and thawing cycles. These cells express several neuronspecific genes, secrete several factors associated with neuroprotection, and exhibit differentiation into neural and glial cells in vitro. In recent years, several studies have documented dopaminergic differentiation of hADSCs.

\section{Introduction}

uman adipose tissue-derived stem cells H (hADSCs) have emerged as a valuable candidate for transplantation therapy of neurodegenerative diseases. These cells express several neuron-specific genes (Soheilifar, Javeri, Amini, \& Taha, 2018), secrete several factors associated with neuroprotection (Lattanzi et al., 2011; Ribeiro et al., 2015), and exhibit differentiation into neural and glial cells in vitro (Feng et al., 2014; Han, Zhang, Song, Liu, Zou, Piao, H., \& Liu, 2014; Safford et al., 2002). In recent years, several studies have documented dopaminergic differentiation of hADSCs (Boroujeni, Gardaneh, Shahriari, Aliaghaei, \& Hasani, 2017; Faghih, Javeri, Amini, \& Taha, 2019; Marei et al., 2018; Singh et al., 2017; Soheilifar et al., 2018) which raises the hope of using these cells for the treatment of Parkinson disease. In our recent work, we successfully induced differentiation of hADSCs to dopaminergic neurons by a growth factor cocktail consisting of sonic hedgehog (SHH), basic fibroblast growth factor (bFGF), fibroblastic growth factor 8 (FGF8), and brainderived neurotrophic factor (BDNF). In that study, TH protein was expressed by a significant proportion of the differentiated cells, and the cells released a detectable amount of dopamine in response to $\mathrm{KCl}$ depolarization (Soheilifar et al., 2018). Also, the differentiated ADSCs expressed GIRK2 but not CALB1, which probably indicates the development of A9-type dopaminergic neurons in our culture settings.

The latter finding is significant for cell therapy of Parkinson disease, where the A9 dopaminergic neurons are the most affected cells (Damier, Hirsch, Agid, \& Gray- biel, 1999), and replenishing them is a critical determining factor for the restoration of patients' motor function (Grealish et al., 2010). However, for transplantation therapy purposes, it is essential to develop highly efficient techniques to generate a more purified population of dopaminergic neurons. One solution may be reprogramming the ADSCs towards a more pluripotent state using different strategies like somatic cell nuclear transfer (Wakayama, Perry, Zuccotti, Johnson, \& Yanagimachi, 1998; Wilmut, Schnieke, McWhir, Kind, \& Campbell, 1997), fusion with pluripotent stem cells (Cowan, Atienza, Melton, \& Eggan, 2005; Tada, Takahama, Abe, Nakatsuji, \& Tada, 2001), coculture with embryonic stem cells (ESCs) (Bahmani, Taha, \& Javeri, 2014), ectopic expression of defined ESC-specific transcription factors (Takahashi et al., 2007; Takahashi \& Yamanaka, 2006), and treatment with the extract of pluripotent cells (Bru et al., 2008; Neri et al., 2007; Rajasingh et al., 2008; Salehi, Foroutan, Javeri, \& Taha, 2017; Taranger et al., 2005; Zhan et al., 2010).

Increasing evidence indicates that transient uptake of regulatory factors from the nuclear or cytoplasmic extracts of fertilized eggs or ESCs reprograms the nuclear function of somatic cells toward pluripotency (Miyamoto et al., 2007; Taranger et al., 2005; Zhan et al., 2010). For this reprogramming event, somatic cells should be reversibly permeabilized and exposed to a cell-free extract of pluripotent cells. This approach has been used to promote morphology, gene expression profile, and growth properties of ESCs in porcine fibroblasts (Miyamoto et al., 2007), NIH3T3 cells (Taranger et al., 2005), and primary rabbit corneal epithelial cells (Zhan et al., 2010). We recently demonstrated that the cytoplasmic extract of mouse ESCs could dedifferentiate hADSCs 
and induce a considerable alteration in the expression of several ESC-associated genes (Salehi et al., 2017).

Despite several studies on the dedifferentiation of somatic cells by the cell-free extracts of pluripotent stem cells (Bru et al., 2008; Neri et al., 2007; Rajasingh et al., 2008; Salehi et al., 2017; Taranger et al., 2005; Zhan et al., 2010), few studies have been conducted on the redifferentiation of this kind of reprogrammed cells (Rajasingh et al., 2008). In the present study, we experienced the reprogramming of hADSCs by the cytoplasmic extract of $\mathrm{hESCs}$ and examined the capability of the reprogrammed cells for differentiation into dopaminergic neurons.

\section{Materials and Methods}

\section{Culture of human ESCs and human ADSCs}

Human ESC line Royan H5 (RSCB0022, Royan Stem Cell Bank, Royan Institute, Tehran, Iran) was cultured on the mitomycin C-inactivated mouse embryonic fibroblasts (MEFs). A medium consisting of DMEM/F12 (Gibco), 15\% KnockOutTM serum replacement (KoSR, Gibco, Life Technologies, USA), 2 mM L-glutamine (Gibco), $0.1 \mathrm{mM}$ non-essential amino acids (SigmaAldrich), $0.1 \mathrm{mM} 2-\beta$-mercaptoethanol (2 $\beta-\mathrm{ME}$, SigmaAldrich) and $4 \mathrm{ng} / \mathrm{mL}$ basic fibroblast growth factor (bFGF) was used to maintain pluripotency of the ESCs and replaced with fresh medium every day.

Subcutaneous abdominal adipose tissue samples were obtained under informed consent from 40 to 50 years old healthy women $(n=5)$ undergoing elective abdominoplasty in Erphan Hospital, Tehran, Iran. The study was approved by the Ethics Committee of the National Institute of Genetic Engineering and Biotechnology (7-8-93/NIGEB).

Adipose tissue-derived stem cells (ADSCs) were isolated and cultured as described previously (Faghih, Javeri, \& Taha, 2017). In brief, adipose tissue samples were digested enzymatically by $2 \mathrm{mg} / \mathrm{mL}$ collagenase I (Thermo Fisher Scientific, Massachusetts, USA) in phosphate-buffered saline (PBS) containing 2\% bovine serum albumin (BSA). After centrifugation, the stromal vascular fraction was resuspended and cultured in a growth medium containing Dulbecco's Modified Eagle's Medium (DMEM), 20\% fetal bovine serum (FBS) and $1 \%$ penicillin and streptomycin (all from Gibco, Thermo Fisher Scientific). The culture medium was renewed daily, and the cells were passaged when expanded to $80 \%-90 \%$ confluency.

\subsection{Cell extract preparation}

Cytoplasmic extract of Royan H1 cells was prepared as described previously for Royan B1 ESCs (Salehi et al., 2017). In brief, the ESCs were isolated from the MEF feeder layer and washed twice with $\mathrm{Ca} 2+, \mathrm{Mg} 2+$-free PBS. After centrifugation, the cells were resuspended in a pre-cold solution consisting of PBS, $1 \mathrm{mM}$ dithiothreitol (DTT), $0.1 \mathrm{mM}$ phenylmethylsulfonyl fluoride (PMSF, Sigma), and $0.1 \mathrm{mM}$ protease inhibitor cocktail (Sigma) and then placed for $1 \mathrm{~h}$ on ice. Next, multiple cycles of freezing in liquid nitrogen and thawing in a water bath were used to lyse the cells, and the cell lysate was centrifuged at $13000 \mathrm{rpm}$. Afterward, ESC extracts were stored in liquid nitrogen. Before reprogramming the ADSCs, the protein concentration of the extract was measured and adjusted to $2 \mathrm{mg} / \mathrm{mL}$.

Permeabilization and reprogramming of the ADSCs

The fourth-passaged ADSCs were isolated by trypsinization and washed in cold Dulbecco's phosphate-buffered saline (DPBS) three times. Then, $5 \times 105$ cells were resuspended in $500 \mu \mathrm{L}$ ice-cold DPBS and incubated at $37^{\circ} \mathrm{C}$ for $2 \mathrm{~min}$. Then, $500 \mathrm{ng} / \mathrm{mL}$ streptolysin O (SLO, Sigma) was added, and the cell suspension was incubated for $50 \mathrm{~min}$ in a $37^{\circ} \mathrm{C}$ water bath with occasional agitation. Afterward, the tube was placed on ice, $500 \mu \mathrm{L}$ cold DPBS was added, and the cells were centrifuged at $1000 \mathrm{rpm}$ for $5 \mathrm{~min}$ at $4^{\circ} \mathrm{C}$.

The permeabilized ADSCs were suspended in $500 \mu \mathrm{L}$ of the ESC extract with an ATP-regenerating system (1 mM ATP, $10 \mathrm{mM}$ creatine phosphate, $25 \mu \mathrm{g} / \mathrm{mL}$ creatine kinase, $100 \mu \mathrm{M}$ GTP, and $1 \mathrm{mM}$ of each nucleotide triphosphate; all from Sigma) and incubated at $37^{\circ} \mathrm{C}$ with occasional agitation for $1 \mathrm{~h}$. Then, the content of the tube was transferred to culture plates with a $2 \mathrm{mM} \mathrm{CaCl} 2$ containing growth medium to reseal the plasma membranes. The next day, the culture medium was renewed, and the reprogrammed cells were cultured for one or two additional weeks (Ext-one week ADSCs and Ext-two weeks ADSCs). The ADSCs, which underwent the same procedure except for incubation in the ADSC extract, were used as the control cells (Cont-ADSCs).

\section{Dopaminergic differentiation of the ADSCs}

The control and reprogrammed ADSCs were seeded at a density of $5 \times 104$ cells $/ \mathrm{mL}$ in $0.1 \%$ gelatin-coated tissue culture plates. The next day, the growth medium was replaced with a dopaminergic induction medium consist- 
Table 1. Primer sets used for gene analysis by RT-PCR and quantitative real-time PCR

\begin{tabular}{ccccc}
\hline Gene & Forward & Reverse & Size & Accession Number \\
\hline OCT4A & GATGTGGTCCGAGTGTGGTT & AAGGGACCGAGGAGTACAGT & 202 & NM_002701 \\
NANOG & GAAGCATCCGACTGTAAAGAATC & TGGTGGAAGAATCAGGGCTGT & 169 & NM_024865 \\
\hline SOX2 & AGAACCCCAAGATGCACAACT & TCCTTCTTCATGAGCGTCTTG & 184 & NM_003106 \\
\hline KLF4 & ATTACCAAGAGCTCATGCCACC & GTGTGCCTTGAGATGGGAACT & 158 & NM-004235 \\
\hline LIN28 & TTCCATGTGCAGCTTACTCT & CAGCAGTTTGCAGGTGGC & 228 & NM_024674 \\
\hline PAX6 & TAGCGAAAAGCAACAGATGGG & CTCCTTCCTGTTGCTGGCA & 155 & NM_000280.3 \\
\hline NES & ACCTCAAGATGTCCCTCAGC & TCAGGACTGGGAGCAAAGATC & 186 & NM_006617 \\
\hline NEFL & CTATGCAGGACACGATCAAC & TTCCAAGAGTTTCCTGTAAGC & 146 & NM_006158 \\
\hline EN1 & GCTATCCTACTTATGGGCTCA & CTCGTTCTTCTTCTTCTTCAGC & 154 & NM_001426 \\
\hline GLI1 & CAATGAGAAGCCGTATGTATGTA & GTAGAAATGGATGGTGCCCG & 162 & NM_005269 \\
\hline LMXB1 & TCACACACGCAGCAGCAGAA & GCAAACAAGACTACCAACAGC & 133 & NM_002316.4 \\
\hline NURR1 & ACTATTCCAGGTTCCAGGCGA & TATGCTAATCGAAGGACAAACAGT & 211 & NM_006186 \\
\hline TH & CAGTTCTCGCAGGACATTGG & TTCACCTCCCCGTTCTGCTTA & 122 & NM_000360 \\
\hline GAPDH & TCCAGCGTACTCCAAAGATTCA & GTCAACTTCAATGTCGGATGGAT & 113 & NM_004048 \\
\hline
\end{tabular}

ing of neurobasal ${ }^{\mathrm{TM}}$ medium (Gibco), 0.5\% B27 supplement (50×, Gibco), $250 \mathrm{ng} / \mathrm{mL}$ SHH, $100 \mathrm{ng} / \mathrm{mL}$ FGF8, and $50 \mathrm{ng} / \mathrm{mL}$ bFGF (all three growth factors from Sigma-Aldrich, St. Louis, USA). Half of the differentiation medium was renewed every three days. On day 9 of differentiation, the medium was supplemented with $50 \mathrm{ng} /$ mL BDNF (Sigma-Aldrich), and the cells were cultured for four additional days.

Gene expression analysis

High pure RNA isolation kit (Roche) and cDNA synthesis kit (Fermentas) were used for total RNA extraction and reverse transcription into cDNA, respectively. PCR amplification on the cDNA templates was done by Taq DNA Polymerase 2x Master Mix Red (Ampliqon A/S, Denmark) and selective primers described in Table 1. $\beta 2$ microglobulin (B2M) and (Glyceraldehyde3-Phosphate Dehydrogenase) GAPDH were used as the internal control genes. The PCR products were detected on $2 \%$ agarose gel.

The expression levels of some genes associated with pluripotency or dopaminergic differentiation were quan- tified by real-time PCR (qPCR). The qPCR experiments were performed using RealQ PCR Master (Ampliqon A/S, Denmark) with Green dye, primers described in Table 1, and four biologic replicates of each group on a Rotor-GeneTM 6000 (Corbett Research, Qiagen) realtime analyzer. B2M and GAPDH were used as internal reference genes to normalize the quantitative data. The data were quantified using REST 2009 (Relative Expression Software Tool, Qiagen) based on Pair Wise Fixed Reallocation Randomization Test $^{\mathrm{R}}$.

\section{Results}

Human ADSCs showing the capability of differentiation into dopaminergic neurons

Undifferentiated ADSCs exhibited a fibroblast-like morphology. We previously characterized the ADSCs based on the expression of mesenchymal stem cell markers and the capability of the cells for adipogenic and osteogenic differentiation (Faghih et al., 2017; Salehi et al., 2017; Soheilifar et al., 2018). After exposure to the dopaminergic induction medium, the ADSCs gradually developed a neuron-like appearance (Figure 1A-D). The differentiated cells expressed NES, NSE, NEFL, GLI1, LMXB1, EN1, 

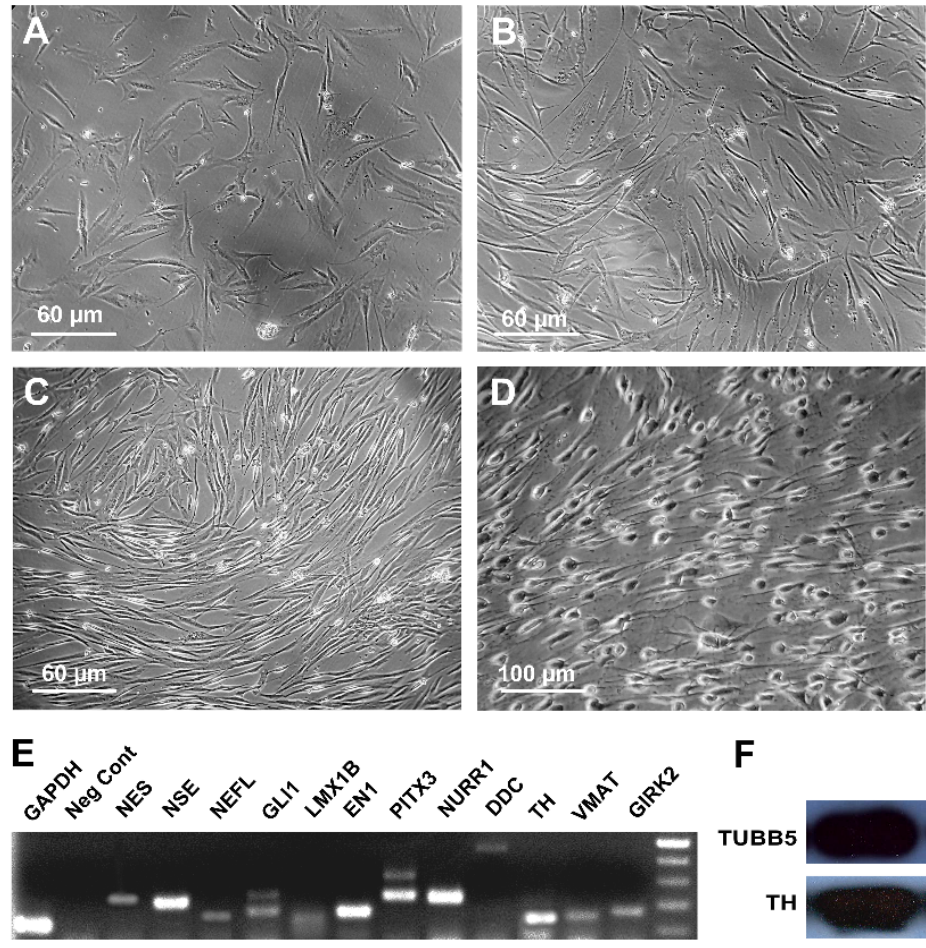

$\mathbf{F}$

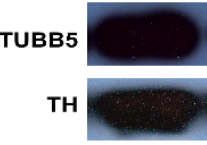

Figure 1. Differentiation of human adipose-derived stem cells (hADSCs) into dopaminergic neurons

A) Fourth-passaged ADSCs just before dopaminergic induction, B-D) The differentiating ADSCs on days 3, 6, and 12 after exposing to dopaminergic induction medium, E) The expression of some neural and dopaminergic-related genes in the differentiated ADSCs, F) The expression of TH protein in the differentiated ADSCs.
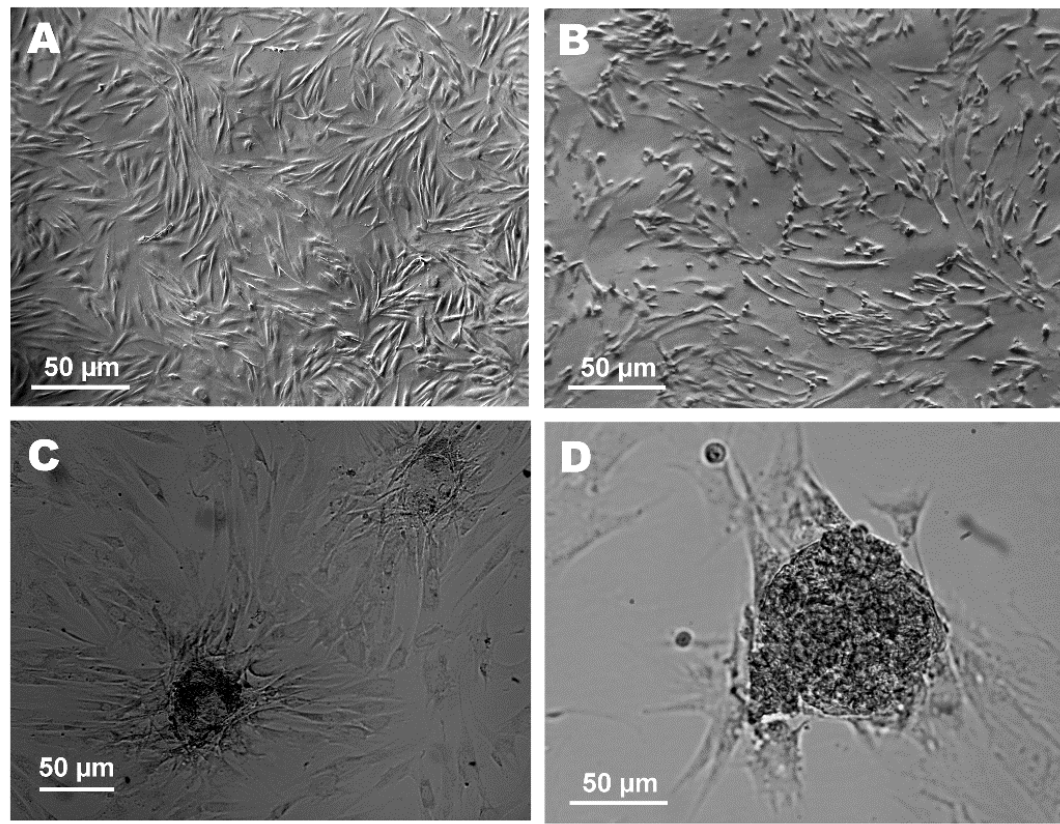

NEUROSCIENCE

Figure 2. Phase contrast images of the control and reprogrammed human adipose tissue-derived stem cells (hADSCs)

A) Fourth-passaged ADSCs, B to D) 24 hours, 4 days and one week after reprogramming of the ADSCs with Human Embryonic Stem Cells (hESC) extract. 


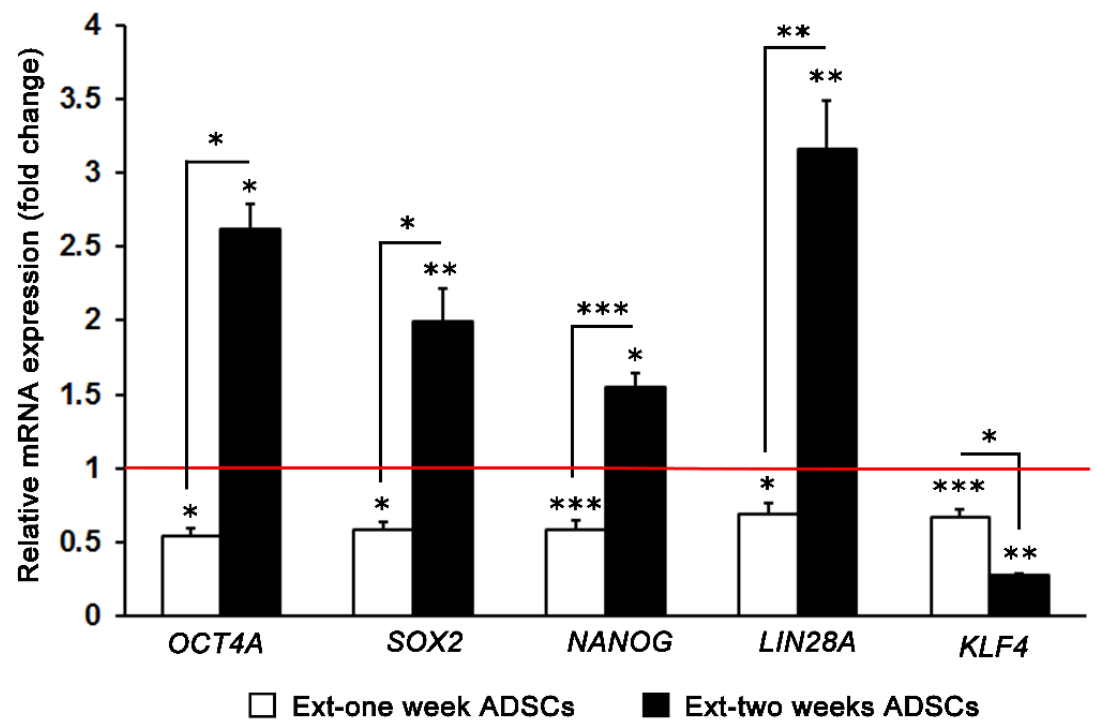

NEUR SCIENCE

Figure 3. Quantitative real-time PCR analysis for the expression of some pluripotency genes in the control and reprogrammed adipose tissue-derived stem cells (ADSCs)

The expression level of each gene in the control ADSCs (indicated by the red line) has been assumed to be 1 , and its expression in the reprogrammed ADSCs has been compared to that. White and black bars, respectively, show the ADSCs 1 week and 2 weeks after treatment with hESC extract. The qPCR experiments were performed with four biologic replicates of each group.

* $\mathrm{P}<0.05$; ** $\mathrm{P}<0.01$; *** $\mathrm{P}<0.001$ (pair wise fixed reallocation randomization test ${ }^{\circledR}$ performed by REST 2009 software).

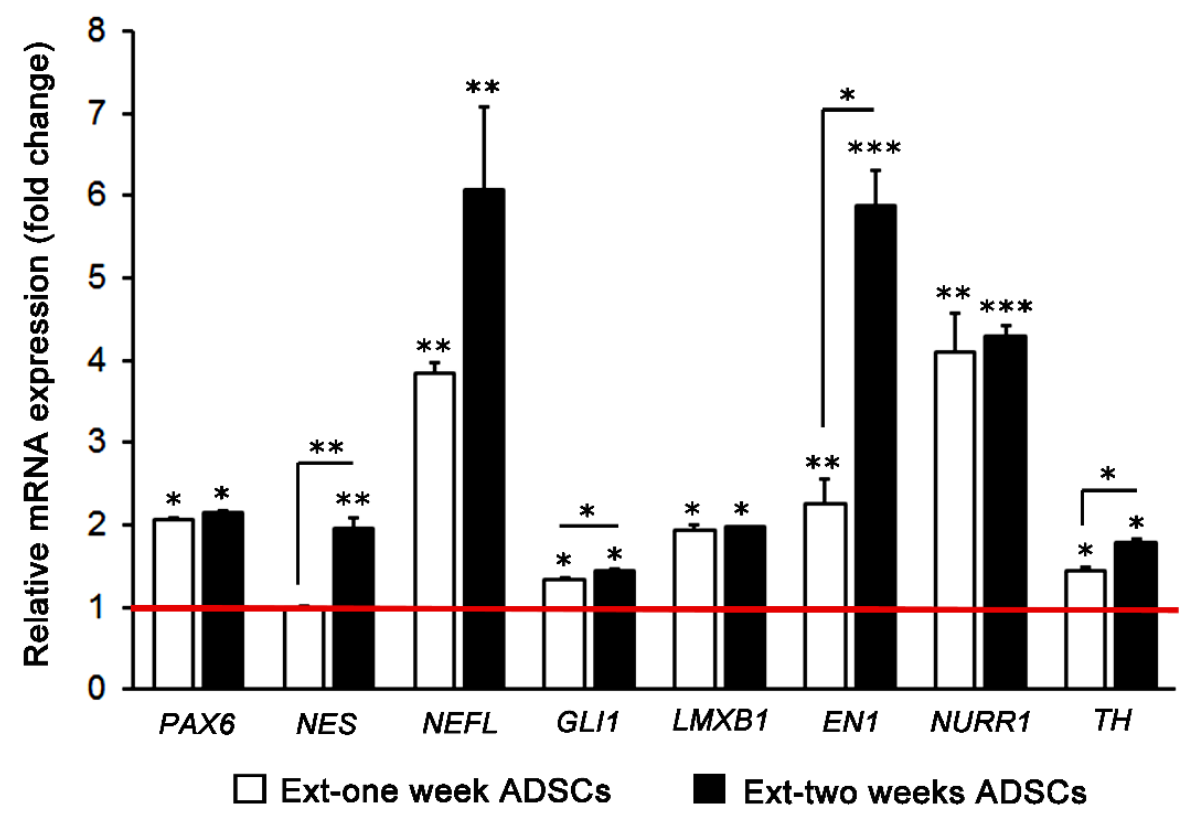

NEUR:SCIENCE

Figure 4. Quantitative real-time PCR analysis for the expression of some neural and dopaminergic-related genes in the differentiated control and reprogrammed Adipose Tissue-Derived Stem Cells (ADSCs)

The expression level of each gene in the differentiated control ADSCs (indicated by the red line) has been assumed to be 1, and its expression in the differentiated reprogrammed ADSCs was compared to that. White and black bars, respectively, show the ADSCs, which were differentiated 1 week and 2 weeks after treatment with ESC extract. The qPCR experiments were performed with four biologic replicates of each group.

${ }^{*} \mathrm{P}<0.05 ;{ }^{* *} \mathrm{P}<0.01$; ${ }^{* *} \mathrm{P}<0.001$ (pair wise fixed reallocation randomization test ${ }^{\circledR}$ performed by REST 2009 software). 
PITX3, NURR1, DDC, TH, VMAT, and GIRK2 mRNAs (Figure 1E). The expression of TH protein was also determined by western blot analysis (Figure 1F).

Treatment of hADSCs with the ESC extract promoting colony formation

The fourth-passaged ADSCs had a fibroblast-like morphology (Figure 1A). Within 1 week after exposure to ESC extract, some colonies were developed in the ADSC cultures (Figure 2B-D). Colony formation was rarely observed in the control or SLO-treated ADSCs.

Treatment of hADSCs with ESC extract and the expression levels of pluripotency genes

As revealed by qPCR analysis, the expression levels of OCT4A, SOX2, NANOG, LIN28A, and KLF4 mRNAs were downregulated in the Ext-one week ADSCs compared to the Cont-ADSCs by mean factors of 0.539 , $0.578,0.586,0.687$ and 0.668 , respectively. Two weeks after reprogramming, the expression of KLF4 further downregulated and reached 0.273 of the Cont-ADSCs, while the expression of OCT4A, SOX2, NANOG, and LIN28A increased by $2.62,2,1.55$, and 3.16 folds, respectively (Figure 3).

Treatment with ESC extract improving dopaminergic differentiation of hADSCs

The qPCR analysis showed that the expression levels of PAX6 and NEFL were upregulated in the differentiated Ext-one week ADSCs compared to the differentiated Cont-ADSCs by mean factors of 2.05 and 3.84, respectively, while the expression of NES did not change significantly. The expression of PAX6, NES, and NEFL mRNAs in the differentiated Ext-two weeks ADSCs was $2.14,1.95$, and 6.08 folds higher than the differentiated Cont-ADSCs, respectively. Moreover, the expression levels of dopaminergic-associated genes of GLI1, LMXB1, EN1, NURR1, and TH, in the differentiated Ext-one week ADSCs were 1.33, 1.94, 2.25, 4.1, and 1.44 folds, and in the differentiated Ext-two weeks ADSCs were 1.45, 1.97, $5.85,4.29$, and 1.77 folds higher than the differentiated Cont-ADSCs, respectively. As a whole, Ext-two weeks ADSCs showed an improved dopaminergic differentiation potential than the other groups (Figure 4).

\section{Discussion}

Up to now, several studies have reported the differentiation of hADSCs into dopaminergic neurons (Boroujeni et al., 2017; Marei et al., 2018; Singh et al., 2017) and the applicability of the ADSCs for the treatment of Parkinson disease (Chi et al., 2018; Choi et al., 2015). We previously used a growth factor cocktail consisting of $\mathrm{SHH}, \mathrm{bFGF}$, FGF8, and BDNF to conduct differentiation of hADSCs toward dopamine-secreting neurons in vitro (Faghih et al., 2019; Soheilifar et al., 2018). Although these reports are promising, techniques to enhance the plasticity of the ADSCs can improve their therapeutic outcome. Reprogramming ADSCs towards a more pluripotent state may be an effective strategy. ESC-specific transcription factors, chromatin remodelers, and small RNAs are three important components of ESC extract which may be involved in this reprogramming event (Taranger et al., 2005).

Previously, Taranger et al. (2005) showed that the extract of undifferentiated embryonic carcinoma cells (ECCs) or ESCs could induce dedifferentiation of somatic cells with restricted plasticity. Based on this experiment, the ESC extracts induced a biphasic wave of OCT4 mRNA and protein expression in 3T3 cells. The first wave, which was detectable one hour after incubation in ESC extracts and persisted for less than $48 \mathrm{~h}$, resulted from the uptake of limited amounts of short-lived OCT4 protein from the extract. However, the second long-lasting wave of OCT4 detected $72 \mathrm{~h}$ after extract removal was due to RNA polymerase II-mediated transcriptional and translational activity of the reprogrammed cells. In the current study, hESC extract was used for reprogramming of hADSCs. Here, we did not assess the expression of OCT4 and other pluripotency genes soon after extract incubation. However, as revealed by qPCR analysis, expressions of OCT4A, SOX2, NANOG, and LIN28A mRNAs were downregulated one week but upregulated two weeks after treatment of the ADSCs with ESC extract (Voutsadakis, 2015). OCT4, SOX2, and NANOG are three key transcription factors that regulate the self-renewal of ESCs (Mitsui et al., 2003; Nichols et al., 1998) and interact with the other transcription factors like KLF4, c-MYC, and LIN28 to maintain pluripotency feature. Our findings demonstrated that more than one week is required to activate the endogenous pluripotency network of the reprogrammed ADSCs and even more time for the transcriptional and translational activation of some genes like KLF4

The reprogrammed ADSC formed several colonies during the first week after exposure to ESC extract. This finding confirms the presence of cell populations with high proliferative capacity. Since cell proliferation and differentiation have a significant inverse relationship, colony formation can also indicate the reprogramming of the ADSCs toward a more dedifferentiated state (Ruijtenberg \& van den Heuvel, 2016).

Dedifferentiation by ESC extract may improve the capability of the ADSCs for directed differentiation. To 
examine this idea, we induced dopaminergic differentiation of the reprogrammed ADSCs using our previously established protocol, i.e., 12-day culture in the serumfree neurobasal medium with a dopaminergic growth factor cocktail (Faghih et al., 2019). Based on the qPCR analysis, reprogramming of the ADSCs by ESC extract upregulated the expression of some neuronal and dopaminergic-associated genes, including PAX6, NES, NEFL, GLI1, LMXB1, EN1, NURR1, and TH. Also, the ADSCs that were differentiated two weeks after reprogramming with ESC extract showed a more noticeable increase in dopaminergic gene expression.

In a study by Han et al. (Han, Sachdev, \& Sidhu, 2010), human fetal fibroblasts were reprogrammed using an ESC extract with $30-35 \mathrm{mg} / \mathrm{mL}$ protein content. Here we applied an ESC extract with $2 \mathrm{mg} / \mathrm{mL}$ protein concentration and did not investigate whether the extracts with higher protein concentrations can affect the duration and degree of ADSC reprogramming. Also, it is important to address the impact of ESC extracts with higher protein contents on the differentiation potential of the ADSCs as well as other mesenchymal stem cell sources.

In conclusion, the findings of the present study demonstrate that the cytoplasmic extract of ESCs contains some regulatory factors which induce the expression of pluripotency-associated markers in hADSCs and that the exposure to ESC extract may serve as a simple and rapid strategy to improve the plasticity of somatic and adult stem cells for cell replacement therapy purposes.

\section{Ethical Considerations}

\section{Compliance with ethical guidelines}

There were no ethical considerations to be considered in this research.

\section{Funding}

This research did not receive any grant from funding agencies in the public, commercial, or non-profit sectors.

\section{Authors' contributions}

All authors equally contributed to preparing this article.

\section{Conflict of interest}

The authors declared no conflict of interest.

\section{Acknowledgments}

This paper is based on the MSc thesis of the first author and is supported by the National Institute of Genetic Engineering and Biotechnology (NIGEB).

\section{References}

Bahmani, L., Taha, M. F., \& Javeri, A. (2014). Coculture with embryonic stem cells improves neural differentiation of adipose tissue-derived stem cells. Neuroscience, 272, 229-239. [DOI:10.1016/j.neuroscience.2014.04.063] [PMID]

Boroujeni, M. E., Gardaneh, M., Shahriari, M. H., Aliaghaei, A., \& Hasani, S. (2017). Synergy between choroid plexus epithelial cell-conditioned medium and knockout serum replacement converts human adipose-derived stem cells to dopamine-secreting neurons. Rejuvenation Research, 20(4), 309-319. [DOI:10.1089/rej.2016.1887] [PMID]

Bru, T., Clarke, C., McGrew, M. J., Sang, H. M., Wilmut, I., \& Blow, J. J. (2008). Rapid induction of pluripotency genes after exposure of human somatic cells to mouse ES cell extracts. Experimental Cell Research, 314(14), 2634-2642. [DOI:10.1016/j. yexcr.2008.05.009] [PMID] [PMCID]

Chi, K., Fu, R. H., Huang, Y. C., Chen, S. Y., Hsu, C. J., \& Lin, S. Z., et al. (2018). Adipose-derived stem cells stimulated with n-butylidenephthalide exhibit therapeutic effects in a mouse model of parkinson's disease. Cell Transplantation, 27(3), 456470. [DOI:10.1177/0963689718757408] [PMID] [PMCID]

Choi, H. S., Kim, H. J., Oh, J. H., Park, H. G., Ra, J. C., \& Chang, K. A., et al. (2015). Therapeutic potentials of human adiposederived stem cells on the mouse model of Parkinson's disease. Neurobiology of Aging, 36(10), 2885-2892. [DOI:10.1016/j. neurobiolaging.2015.06.022] [PMID]

Cowan, C. A., Atienza, J., Melton, D. A., \& Eggan, K. (2005). Nuclear reprogramming of somatic cells after fusion with human embryonic stem cells. Science, 309(5739), 1369-1373. [DOI:10.1126/science.1116447] [PMID]

Damier, P., Hirsch, E. C., Agid, Y., \& Graybiel, A. M. (1999). The substantia nigra of the human brain. II. Patterns of loss of dopamine-containing neurons in Parkinson's disease. Brain, 122(Pt 8), 1437-1448. [DOI:10.1093/brain/122.8.1437] [PMID]

Faghih, H., Javeri, A., Amini, H., \& Taha, M. F. (2019). Directed differentiation of human adipose tissue-derived stem cells to dopaminergic neurons in low-serum and serum-free conditions. Neuroscience Letter, 708, 134353. [DOI:10.1016/j.neulet.2019.134353] [PMID]

Faghih, H., Javeri, A., \& Taha, M. F. (2017). Impact of early subcultures on stemness, migration and angiogenic potential of adipose tissue-derived stem cells and their resistance to in vitro ischemic condition. Cytotechnology, 69(6), 885-900. [DOI:10.1007/s10616-017-0104-5] [PMID] [PMCID]

Feng, N., Han, Q., Li, J., Wang, S., Li, H., \& Yao, X., et al. (2014). Generation of highly purified neural stem cells from human adipose-derived mesenchymal stem cells by Sox1 activation. 
Stem Cells and Development, 23(5), 515-529. [DOI:10.1089/ scd.2013.0263] [PMID] [PMCID]

Grealish, S., Jonsson, M. E., Li, M., Kirik, D., Bjorklund, A., \& Thompson, L. H. (2010). The A9 dopamine neuron component in grafts of ventral mesencephalon is an important determinant for recovery of motor function in a rat model of Parkinson's disease. Brain, 133(Pt 2), 482-495. [DOI:10.1093/ brain/awp328] [PMID] [PMCID]

Han, C., Zhang, L., Song, L., Liu, Y., Zou, W., Piao, H., \& Liu, J. (2014). Human adipose-derived mesenchymal stem cells: A better cell source for nervous system regeneration. Chinese Medical Journal, 127(2), 329-337. [PMID]

Han, J., Sachdev, P. S., \& Sidhu, K. S. (2010). A combined epigenetic and non-genetic approach for reprogramming human somatic cells. PLoS One, 5(8), e12297. [DOI:10.1371/journal. pone.0012297] [PMID] [PMCID]

Lattanzi, W., Geloso, M. C., Saulnier, N., Giannetti, S., Puglisi, M. A., Corvino, V., et al. (2011). Neurotrophic features of human adipose tissue-derived stromal cells: In vitro and in vivo studies. Journal of Biomedicine \& Biotechnology, 2011, 468705. [DOI:10.1155/2011/468705] [PMID] [PMCID]

Marei, H. E. S., El-Gamal, A., Althani, A., Afifi, N., Abd-Elmaksoud, A., Farag, A., et al. (2018). Cholinergic and dopaminergic neuronal differentiation of human adipose tissue derived mesenchymal stem cells. Journal of Cellular Physiology, 233(2), 936-945. [DOI:10.1002/jcp.25937] [PMID]

Mitsui, K., Tokuzawa, Y., Itoh, H., Segawa, K., Murakami, M., Takahashi, K., et al. (2003). The homeoprotein Nanog is required for maintenance of pluripotency in mouse epiblast and ES cells. Cell, 113(5), 631-642. [DOI:10.1016/S00928674(03)00393-3]

Miyamoto, K., Furusawa, T., Ohnuki, M., Goel, S., Tokunaga, T., Minami, N., et al. (2007). Reprogramming events of mammalian somatic cells induced by Xenopus laevis egg extracts. Molecular Reproduction and Development, 74(10), 1268-1277. [DOI:10.1002/mrd.20691] [PMID]

Neri, T., Monti, M., Rebuzzini, P., Merico, V., Garagna, S., \& Redi, C. A., et al. (2007). Mouse fibroblasts are reprogrammed to Oct- 4 and Rex-1 gene expression and alkaline phosphatase activity by embryonic stem cell extracts. Cloning and Stem Cells, 9(3), 394-406. [DOI:10.1089/clo.2006.0011] [PMID]

Nichols, J., Zevnik, B., Anastassiadis, K., Niwa, H., Klewe-Nebenius, D., Chambers, I., et al. (1998). Formation of pluripotent stem cells in the mammalian embryo depends on the POU transcription factor Oct4. Cell, 95(3), 379-391. [DOI:10.1016/ S0092-8674(00)81769-9]

Rajasingh, J., Lambers, E., Hamada, H., Bord, E., Thorne, T., \& Goukassian, I., et al. (2008). Cell-free embryonic stem cell extract-mediated derivation of multipotent stem cells from NIH3T3 fibroblasts for functional and anatomical ischemic tissue repair. Circulation Research, 102(11), e107-117. [DOI:10.1161/CIRCRESAHA.108.176115] [PMID] [PMCID]

Ribeiro, T. B., Duarte, A. S. S., Longhini, A. L. F., Pradella, F., \& Farias, A. S., et al. (2015). Neuroprotection and immunomodulation by xenografted human mesenchymal stem cells following spinal cord ventral root avulsion. Scientific Reports, 5, 16167. [DOI:10.1038/srep16167] [PMID] [PMCID]

Ruijtenberg, S., \& van den Heuvel, S. (2016). Coordinating cell proliferation and differentiation: Antagonism between cell cycle regulators and cell type-specific gene expression. Cell Cycle, 15(2), 196-212. [DOI:10.1080/15384101.2015.1120925] [PMID] [PMCID]

Safford, K. M., Hicok, K. C., Safford, S. D., Halvorsen, Y. D., Wilkison, W. O., Gimble, J. M., \& Rice, H. E. (2002). Neurogenic differentiation of murine and human adipose-derived stromal cells. Biochemical and Biophysical Research Communications, 294(2), 371-379. [DOI:10.1016/S0006-291X(02)00469-2]

Salehi, P. M., Foroutan, T., Javeri, A., \& Taha, M. F. (2017). Extract of mouse embryonic stem cells induces the expression of pluripotency genes in human adipose tissue-derived stem cells. Iranian Journal of Basic Medical Sciences, 20(11), 1200-1206. [DOI:10.22038/IJBMS.2017.9464] [PMID] [PMCID]

Singh, M., Kakkar, A., Sharma, R., Kharbanda, O. P., Monga, N., Kumar, M., et al. (2017). Synergistic effect of BDNF and FGF2 in efficient generation of functional dopaminergic neurons from human mesenchymal stem cells. Scientific Reports, 7(1), 10378. [DOI:10.1038/s41598-017-11028-z] [PMID] [PMCID]

Soheilifar, M. H., Javeri, A., Amini, H., \& Taha, M. F. (2018). Generation of dopamine-secreting cells from human adipose tissue-derived stem cells in vitro. Rejuvenation Reserch, 21(4), 360-368. [DOI:10.1089/rej.2017.1994] [PMID]

Tada, M., Takahama, Y., Abe, K., Nakatsuji, N., \& Tada, T. (2001). Nuclear reprogramming of somatic cells by in vitro hybridization with ES cells. Current Biology, 11(19), 1553-1558. [DOI:10.1016/S0960-9822(01)00459-6]

Takahashi, K., Tanabe, K., Ohnuki, M., Narita, M., Ichisaka, T., Tomoda, K., \& Yamanaka, S. (2007). Induction of pluripotent stem cells from adult human fibroblasts by defined factors. Cell, 131(5), 861-872. [DOI:10.1016/j.cell.2007.11.019] [PMID]

Takahashi, K., \& Yamanaka, S. (2006). Induction of pluripotent stem cells from mouse embryonic and adult fibroblast cultures by defined factors. Cell, 126(4), 663-676. [DOI:10.1016/j. cell.2006.07.024] [PMID]

Taranger, C. K., Noer, A., Sorensen, A. L., Hakelien, A. M. Boquest, A. C., \& Collas, P. (2005). Induction of dedifferentiation, genomewide transcriptional programming, and epigenetic reprogramming by extracts of carcinoma and embryonic stem cells. Molecular Biology of the Cell, 16(12), 5719-5735. [DOI:10.1091/mbc.e05-06-0572] [PMID] [PMCID]

Voutsadakis, I. A. (2015). The network of pluripotency, epithelial-mesenchymal transition, and prognosis of breast cancer Breast Cancer: Targets and Therapy, 7, 303-319. [DOI:10.2147/ BCTT.S71163] [PMID] [PMCID]

Wakayama, T., Perry, A. C., Zuccotti, M., Johnson, K. R., \& Yanagimachi, R. (1998). Full-term development of mice from enucleated oocytes injected with cumulus cell nuclei. Nature, 394(6691), 369-374. [DOI:10.1038/28615] [PMID]

Wilmut, I., Schnieke, A. E., McWhir, J., Kind, A. J., \& Campbell, K. H. (1997). Viable offspring derived from fetal and adult mammalian cells. Nature, 385(6619), 810-813. [DOI:10.1038/385810a0] [PMID]

Zhan, W., Liu, Z., Liu, Y., Ke, Q., Ding, Y., \& Lu, X., et al. (2010). Modulation of rabbit corneal epithelial cells fate using embryonic stem cell extract. Molecular Viston, 16, 1154-1161. https:/ / www.scienceopen.com/.pdf 
